\title{
Elucidation of mechanisms of actions of thymoquinone-enriched methanolic and volatile oil extracts from Nigella sativa against cardiovascular risk parameters in experimental hyperlipidemia
}

\author{
Shafeeque Ahmad ${ }^{*}$ and Zafarul H Beg
}

\begin{abstract}
Background: Nigella sativa belonging to the Ranunculaceae family has been reported to use for thousands of years as protective and curative traditional medicine against a number of diseases. GC-MS analysis of methanolic extract (ME) and volatile oil (VO) extracted from Nigella sativa seed oil was performed by two different mass spectrometry libraries, WIIEY8 and NIST05s. The cholesterol lowering and antioxidant actions of VO and ME fractions were investigated in atherogenic suspension fed rats.
\end{abstract}

Methods: In this study, four groups of male Wistar rats were used: normolipidemic control (NLP-C), hyperlipidemic control (HLP-C), methanolic extract (HLP-ME) and volatile oil treated (HLP-VO) groups for 30 days of duration. P value $<0.05$ was assumed as significant data in groups.

Results: Administration of atherogenic suspension to male Wistar rats for 30 days resulted in a marked increase of plasma triglycerides and total cholesterol, and significant change in plasma lipoprotein levels along with a decrease in antioxidant arylesterase activity in hyperlipidemic control (HLP-C) group. The oral feeding of $100 \mathrm{mg}$ ME or 20 mg VO per rat/day effectively reduced the plasma triglycerides to near normal level, while high density lipoprotein cholesterol and its subfraction along with arylesterase activity levels were significantly increased. The test fractions elicited a significant decrease in hepatic HMG-CoA reductase activity. The fractions significantly blocked the ex vivo basal and in vitro maximal formation of conjugated diene and malondialdehyde, and lengthened the lag times of low density lipoprotein, small dense low density lipoprotein and large buoyant low density lipoprotein. ME possessing $\omega-6$ linoleic acid along with palmitic acid active compounds was more effective than VO extract containing thymol and isothymol phenolic antioxidant compounds, thymoquinone phenolic compound common to the both extracts, via reduction in hepatic HMG-CoA reductase activity as well as antioxidant mechanisms.

Conclusion: The both extracts especially, ME significantly improve cardiovascular risk parameters in treated rats, and can be used in reactive oxygen species disorders such as cardiovascular diseases.

Keywords: Nigella sativa, Methanolic extract, Volatile oil, GC-MS, HMG-CoA reductase, Lipoprotein oxidation

\footnotetext{
* Correspondence: azamshafeeque1@gmail.com

Department of Biochemistry, Jawahar Lal Nehru Medical College, Aligarh Muslim University, Aligarh, UP 202 002, India
} 


\section{Background}

The World Health Organization (WHO) has declared that due to cardiovascular disease (CVD), the death toll of people will reach approximately 23.6 million around the world by 2030 [1]. It is well known that hypercholesterolemic patients are more prone to heart attack than normal blood lipid profiles. According to the WHO report, consumption of unhealthy diets, rich in fat, salt and free sugar, and low in complex carbohydrates, fruits and vegetables are responsible for CVD [2]. Diets rich in cholesterol produce free radicals, followed by oxidative stress and hypercholesterolemia [3,4]. Oxidative stress is one of the factors that causes rise in the level of blood cholesterol and it may result in atherogenesis [5]. High concentration of LDL-C in plasma is one of the major causes of coronary heart disease (CHD) as evidenced by epidemiological studies [6]. In comparison to lb-LDL, sd-LDL is highly atherogenic because of the presence of properties like enhanced susceptibility to oxidation, higher penetration in the arterial wall, lower binding affinity to the LDL-receptor, and prolonged plasma half life $[7,8]$. Hence, it will be focusing attention on sd-LDL as a new precise and useful CHD marker [9-11]. Blood cholesterol is recommended to be within normal range to preserve normal cell functions. At present, there are several methods known to control blood cholesterol levels, including dietary fats, bile acids sequestering agents and use of HMG-CoA reductase inhibitors. Among them, HMG-CoA reductase is the key enzyme in the cholesterol biosynthesis pathway that could be an important target of an inhibitor for managing blood cholesterol levels [12].

Use of the modern drugs explores various drawbacks that are expensive, beyond the reach of a majority of the population of the world and multiple side effects. Therefore, today drugs from indigenous sources should be designed and suitable different combinations of such drugs tested for therapeutic purposes, which posses minimal adverse effects and multiple targets in preventing and curing diseases. A number of epidemiological studies have been reported that plant foods carry a high quantity of phenolic compounds, are effective against fatal chronic diseases like cancer, neurodegenerative and CVD [13-15]. Such compounds show antioxidant properties. Their free radical scavenging properties solely rely upon constituents as well as synergistic interactions [16]. Various types of reactive oxygen species (ROS) are generated in our body and their removal must be continually done for the maintenance of normal cellular functions. Phenolic compounds $(\mathrm{POH})$ deactivate various types of free radicals such as hydroxyl radicals and peroxyl radicals $\left(\mathrm{R}^{*}\right)$ by donating hydrogen, which interrupts chain reactions:

$$
\begin{aligned}
& \mathrm{R}^{*}+\mathrm{POH} \rightarrow \mathrm{R}-\mathrm{H}+\mathrm{PO}^{*}(\text { Interruption reaction }) \\
& \mathrm{PO}^{*}+\mathrm{PO} \rightarrow \mathrm{PO}-\mathrm{OP}(\text { Stabilization reaction })
\end{aligned}
$$

In the above reactions, stabilization of phenoxyl radicals $\left(\mathrm{PO}^{*}\right)$ is possible through resonance and/or intramolecular hydrogen bonding [17]. Thus, consumption of phenolic compounds may be useful to the health. Recently, Nigella sativa (NS) has been reported to possess multiple health beneficial properties. Several studies have been reported that methanolic extract (ME) [14], volatile oil (VO) [18] from black cumin and their principal active constituent thymoquinone [19], show antioxidant properties. Phenolic compounds such as thymol, isothymol have been shown to have antioxidant actions [20], and essential fatty acid, $\omega-6$ linoleic acid [21] or $\omega-6$ linoleic acid with palmitic acid have hypolipidemic property [22].

Elevated levels of TG and TG rich VLDL, these two parameters are regarded as risk factors for atherosclerosis $[23,24]$, while an elevated level of plasma HDL-C is considered beneficial to the health because these are inversely correlated with the risk factor for atherosclerosis [25]. In agreement with these concepts, drugs that cause reduction of TG, TG rich VLDL and increase in HDL would be helpful in decrease in the morbidity and mortality from CAD $[25,26]$. HDL is assumed a key lipoprotein that helps lipid removal from macrophage foam cells in the arterial wall, and thus protects LDL oxidation. Several studies have been performed using albino Wistar rats as experimental atherosclerosis model, and they found it suitable and effective model to be used in condition of hypercholesterolemia and atherosclerosis $[27,28]$. In the present study, we have performed gas chromatography (GC) analysis of the ME and VO, fractionated from NS seed oil and identified their constituents by using two different mass spectrometry (MS) libraries. In order to understand the mechanisms of lipid lowering actions of these test fractions, we explored the hepatic HMG-CoA reductase activity as well as their protective effect on HDL-linked arylesterase activity, in rats for 30 days. Further, the antioxidant protection of the test fractions afforded to LDL and its density subfractions, sd-LDL and lb-LDL, from in vivo and $\mathrm{Cu}^{2+}$-catalyzed in vitro oxidation was investigated. This objective was achieved by measuring ex vivo basal and in vitro maximal $\mathrm{CD}$ formations and lag times, as well as ex vivo basal and maximal production of MDA in LDL, sd-LDL and lb-LDL lipoproteins isolated from plasma of experimental animals.

\section{Materials and methods Materials}

Export quality, edible Nigella sativa (NS) seed oil, was bought from a local store. Phenyl acetate was procured from Sigma-Aldrich Inc. USA. Highly purified thiobarbituric acid and hydroxylamine hydrochloride were purchased from HiMedia Laboratories Pvt. Ltd. (Mumbai, India), while phosphotungstic acid was bought from Loba Chemie (Mumbai, India). And HMG-CoA was 
procured from Pharmacia, USA. Mevalonolactone was purchased from United States Bioch. Corp., USA, while sodium dodecyl sulfate was obtained from Bio-Rad Laboratories USA. Triglycerides assay kit for determination of triglycerides was procured from Autospan Kit SPAN Diagnostics (New Delhi, India). Other chemicals and reagents were used in this research of analytical grade.

\section{Preparation of methanolic extract (ME) and volatile oil (VO) extracts from NS seed oil}

The ME fraction, used in the present study, was extracted by stirring $10 \mathrm{~g}$ of the NS seed oil with $100 \mathrm{ml}$ of methanol for $100 \mathrm{~min}$ at ambient temperature. The methanol layer was removed, and then evaporated at $45^{\circ} \mathrm{C}$. The VO fraction was extracted from NS seed oil by steam distillation. The procedure is essentially the same as described for the isolation of VO extract from NS seeds [29] with minor modification. Briefly, $400 \mathrm{ml}$ of distilled water was added to a distillation flask containing $12 \mathrm{~g}$ of NS seed oil, and the temperature was set to the boiling point. The process of distillation was continued until about $150 \mathrm{ml}$ of the distillate containing $\mathrm{VO}$ was collected in a dark glass bottle. The distillate was extracted with $50 \mathrm{ml}$ of diethyl ether, moisture was removed by the addition of anhydrous sodium sulphate and the resultant ether extract was evaporated at $40^{\circ} \mathrm{C}$. The $\mathrm{ME}$ and $\mathrm{VO}$ extracts thus obtained were stored under nitrogen in dark colored bottles at $4^{\circ} \mathrm{C}$.

\section{GC-MS analysis}

GC-MS analyses of ME and VO extracted from NS oil were performed by GCMS (QP2010 plus system Shimadzu). Separation was carried out at chromatograph column of SLB60m $\times$ ID $0.25 \mathrm{~mm} \times$ thickness $0.25 \mu \mathrm{m}$. Column oven temperature was initially set at $70^{\circ} \mathrm{C}$ for $2 \mathrm{~min}$, which was increased to $310^{\circ} \mathrm{C}$ at the rate of $5^{\circ} \mathrm{C}$ per min with $20 \mathrm{~min}$ hold. The carrier gas, helium was used with a flow of 1.21 $\mathrm{ml}$ per min. The split ratio was adjusted to 30.0. Injector temperature was adjusted at $270^{\circ} \mathrm{C}$. Ionization voltage was $70 \mathrm{eV}$. The constituents present in the samples were identified by matching their mass spectra with two mass spectra libraries, namely WIIEY8.LIB and NIST05s.LIB. The software of the system determined relative percentage amount of the compounds that exist in the both samples.

\section{Animals and treatments}

The Board of Studies of the Biochemistry Department and the Animal Ethics Committee of J.N. Medical College, A.M.U., Aligarh, where the present research work was performed, approved the experimental study. Healthy male Wistar albino rats, weighting 180-210 g from inbred colony maintained by central animal facility of J.N. Medical College, were allowed free access to standard rat chow and water. Ten percent ME and 2\% VO suspensions were prepared by dissolving in DMSO (12.5\%) and then homogenized with saline. The rats were divided into the following groups:

Normolipidemic control (NLP-C): This group of animals containing five rats was orally administered $1 \mathrm{ml}$ solution of saline containing $12.5 \%$ DMSO, twice $0.5 \mathrm{ml}$ each in the morning and evening per rat per day for 30 days of duration.

Hyperlipidemic control (HLP-C): For the induction of hyperlipidemia, before the administration of $1.0 \mathrm{ml}$ atherogenic suspension, this experimental group received $1.0 \mathrm{ml}$ of saline containing $12.5 \%$ DMSO, twice $0.5 \mathrm{ml}$ each in the morning and evening per rat per day for 30 days.

Hyperlipidemic methanolic extract (HLP-ME): One milliliter of $10 \% \mathrm{ME}$ suspension was given to the rats, before the administration of $1.0 \mathrm{ml}$ of atherogenic suspension, twice $0.5 \mathrm{ml}$ each in the morning and evening per rat per day for 30 days.

Hyperlipidemic volatile oil (HLP-VO): One milliliter of $2 \%$ VO suspension was administered, before the administration of $1.0 \mathrm{ml}$ of atherogenic suspension, twice $0.5 \mathrm{ml}$ each in the morning and evening per rat per day for 30 days.

As in previous studies, ME and VO were taken at dose of $810 \mathrm{mg}$ and $400 \mathrm{mg} /$ day/ $/ \mathrm{kg}$ body weight of male Wistar rats, respectively $[30,31]$. The $\mathrm{LD}_{50}$ of $\mathrm{ME}$ was not found at four different doses, 6, 9, 14 and $21 \mathrm{~g} / \mathrm{kg}$, and further no hepatic toxicity of ME at $6 \mathrm{~g} /$ day $/ \mathrm{kg}$ body weight of mice was reported [32], and the $\mathrm{LD}_{50}$ of $\mathrm{VO}$ was found to be $0.5 \mathrm{ml} /$ day $/ \mathrm{kg}$ body weight of male albino mice [33]. So based on these reports, we took single, safe and effective dose of $\mathrm{ME}$ and VO.

\section{Plasma separation from blood}

At the end of the experiment, rats were anaesthetized and blood was taken out by cardiac puncture from overnight fasted rats in each group. The blood was collected in heparinized tubes. After incubation at $4{ }^{\circ} \mathrm{C}$ for $2-3 \mathrm{~h}$, blood samples were centrifuged at 2,500 rpm for $30 \mathrm{~min}$. Plasma was aliquoted and stored at $4^{\circ} \mathrm{C}$ for future use.

\section{Low density lipoprotein (LDL), small dense (sd)-LDL and large buoyant (Ib)-LDL fractionation}

Basically the precipitation method for plasma LDL isolation as described by Wieland and Seidel [34] was employed. Briefly, the precipitation buffer consisted of $64 \mathrm{mM}$ trisodium citrate, $\mathrm{pH} 5.05$, plus 50,000 IU/L heparin. Prior to LDL precipitation, plasma samples and precipitation reagent were allowed to equilibrate to room temperature. Then $1.0 \mathrm{ml}$ of plasma was added to $7.0 \mathrm{ml}$ of heparincitrate buffer, mixed properly, and then it was allowed to stand for $10 \mathrm{~min}$ at $22^{\circ} \mathrm{C}$. Then it was centrifuged at 1,500 rpm for $10 \mathrm{~min}$ at $22^{\circ} \mathrm{C}$. The pellet thus obtained was resuspended in $1.0 \mathrm{ml}$ of $0.1 \mathrm{M}$ sodium phosphate buffer saline, $\mathrm{pH}$ 7.4. Hirano et al. [10] described the isolation of 
small dense (sd)-LDL and large buoyant (lb)-LDL from isolated LDL which is based on the two-step procedure quantifying sd-LDL from serum by heparin- $\mathrm{Mg}^{2+}$ precipitation. In our modified method for the isolation of sd-LDL and lb-LDL from plasma LDL, $0.1 \mathrm{ml}$ precipitation reagent solution prepared by taking 15 IU of heparin and $90 \mathrm{mM} \mathrm{MgCl}$ was added to $0.1 \mathrm{ml}$ of LDL. The sample was properly mixed, then incubated at $37^{\circ} \mathrm{C}$ for $10 \mathrm{~min}$. Each sample was then incubated in an ice-bath for $15 \mathrm{~min}$. Finally, each sample was centrifuged at 10,000 rpm for $15 \mathrm{~min}$ at $4^{\circ} \mathrm{C}$. The upper supernatant and lower portions of the samples containing sd-LDL and lb-LDL, respectively were carefully removed and saved. The lb-LDL fraction as pellet was dissolved in $0.1 \mathrm{M}$ sodium phosphate buffer saline, $\mathrm{pH}$ 7.4. Appropriate quantities of LDL, sd-LDL and lb-LDL fractions were taken out for further analysis.

Isolation of plasma very low density lipoprotein (VLDL) Plasma VLDL was isolated as described by Bachorik and Albers [35]. Briefly, for the isolation of VLDL, 0.075 volume of $10 \%$ sodium dodecyl sulfate solution was added to 1 volume of plasma, and then properly mixed. Afterwards, it was incubated for $2 \mathrm{~h}$ at $37^{\circ} \mathrm{C}$ and finally centrifuged at $10,000 \mathrm{rpm}$ for 10 minutes at room temperature. At the top of the centrifuge tube, VLDL pellicle was appeared. The supernatant was carefully removed. The residue of VLDL pellicle was dissolved in one volume of $1 \%$ sodium dodecyl sulfate by warming at $37^{\circ} \mathrm{C}$.

\section{Isolation of plasma high density lipoprotein cholesterol (HDL-C) and $\mathrm{HDL}_{3}-\mathrm{C}$ subfraction}

High density lipoprotein cholesterol from plasma was isolated by the method of Kostner [36] and $\mathrm{HDL}_{3}-\mathrm{C}$ subfraction of HDL-C was isolated by the procedure as described by Bachorik and Albers [35]. In brief, 0.2 volume of phosphotungstic acid and magnesium chloride (1:1) was added to 1 volume of plasma, and then instantly and properly mixed. It was finally centrifuged for $10 \mathrm{~min}$ at 12,000 rpm at room temperature. The clear supernatant was carefully removed and thus represented the plasma HDL-C. For HDL-C fractionation, reagent ' $\mathrm{X}$ ' was prepared by mixing 1 volume of $40 \mathrm{~g} / \mathrm{L}$ dextran sulfate, $\mathrm{Ph} 7.0$ with 3 volumes of $2 \mathrm{M} \mathrm{MgCl}_{2} \cdot 6 \mathrm{H}_{2} \mathrm{O}, \mathrm{Ph} 7.0$. To fractionate HDL-C, 0.1 volume of reagent ' $\mathrm{X}$ ' was added to one volume of HDL-C and mixed immediately. Then it was centrifuged at $5,000 \mathrm{rpm}$ for $30 \mathrm{~min}$ at $4^{\circ} \mathrm{C}$. Aliquots of clear supernatant were used for $\mathrm{HDL}_{3}-\mathrm{C}$ analysis. $\mathrm{HDL}_{2}-\mathrm{C}$ was then calculated as the difference between HDL-C and $\mathrm{HDL}_{3}-\mathrm{C}$ concentration.

\section{Determination of plasma and lipoprotein cholesterols} Plasma total cholesterol, VLDL, LDL and HDL lipoprotein cholesterols and their fraction cholesterols were determined as previously stated by Ahmad and Beg [37] in detail.

\section{Determination of plasma triglycerides}

Triglycerides present in plasma were determined by enzymatic kit, based on a modified Trinder color reaction, which produces fast, linear, and end point reaction [38]. The intensity of the color produced of the triglycerides in the plasma samples was measured at $500 \mathrm{~nm}$ in a Beckman DU 640 spectrophotometer. Triolein was used as standard to calculate triglycerides concentration in plasma samples.

\section{Determination of hepatic HMG-CoA reductase activity}

HMG-CoA reductase enzyme activity in liver homogenate was assayed indirectly by employing the method of Rao and Ramakrishnan [39]. Briefly, fresh 10 g liver was homogenized in $90 \mathrm{ml}$ of saline arsenate. Then equal volume of $10 \%$ liver homogenate and $5 \%$ perchloric acid (5 ml each) was taken out and thoroughly mixed, and kept for $5 \mathrm{~min}$ at room temperature. Afterwards, it was centrifuged at 2,000 rpm for $10 \mathrm{~min}$. Half milliliter of freshly prepared $1 \mathrm{M}$ aqueous hydroxylamine hydrochloride was mixed with one $\mathrm{ml}$ of the supernatant obtained after centrifugation. For determination of HMG-CoA reductase, $0.5 \mathrm{ml}$ of alkaline hydroxylamine hydrochloride was added and properly mixed. Then it was allowed to incubate for $5 \mathrm{~min}$ at room temperature. Afterwards, $1.5 \mathrm{ml}$ of $0.616 \mathrm{M}$ ferric chloride solution consisted of $5.2 \%$ trichloroacetic acid prepared in 0.65 $\mathrm{N} \mathrm{HCl}$ was added to the above sample and thoroughly mixed, and incubated for $10 \mathrm{~min}$ at room temperature. Absorbance was recorded at $540 \mathrm{~nm}$ against a reagent blank in a Beckman DU 640 spectrophotometer.

\section{Determination of plasma arylesterase antioxidant enzyme activity}

Plasma arylesterase antioxidant enzyme activity was essentially assayed by the method of Ayub et al. [40]. Phenyl acetate was used as the substrate. The reaction mixture consisted of $100 \mathrm{mM}$ tris buffer, $\mathrm{pH} 8.0,1 \mathrm{mM}$ $\mathrm{CaCl}_{2}, 1 \mathrm{mM}$ phenyl acetate and suitable aliquots of plasma sample. After 5 min of preincubation period of the samples, the reaction was started by adding the phenyl acetate and incubated for different time intervals at $25^{\circ} \mathrm{C}$. Absorbance of initial rates of hydrolysis was determined spectrophotometrically at $270 \mathrm{~nm}$ against a reagent blank, while nonenzymatic hydrolysis of phenyl acetate was subtracted from the total rate of hydrolysis. The molar extinction coefficient of $1.31 \times 10^{3} \mathrm{M}^{-1} \mathrm{~cm}^{-1}$ was used to calculate the arylesterase enzyme activity in the plasma sample [41]. 


\section{Determination of ex vivo and in vitro $\mathrm{Cu}^{2+}$-mediated susceptibility of LDL, sd-LDL and Ib-LDL to oxidation} The ex vivo and in vitro $\mathrm{Cu}^{2+}$-mediated susceptibility of LDL, sd-LDL and lb-LDL to oxidation was determined by as described by Esterbauer et al. [42] and Esterbauer et al. [43]. Before oxidation studies of lipoprotein fractions by determining the lag phase of conjugated diene formation were dialyzed against $10 \mathrm{mM}$ phosphate buffer saline (PBS), $\mathrm{pH} 7.4$, for $12 \mathrm{~h}$. At different time of interval of oxidation, $100 \mu \mathrm{g}$ of LDL, sd-LDL or lb-LDL dissolved in 1.0 $\mathrm{ml}$ of PBS was added to $0.5 \mathrm{mM}$ EDTA, and then mixed, afterward stored at $4^{\circ} \mathrm{C}$ and used for the assessment of conjugated diene formation. In another set of zero time ex vivo oxidation, one $\mathrm{ml}$ aliquot of each sample was added to a tube carrying $0.5 \mathrm{mM}$ EDTA, $\mathrm{pH}$ 7.4, for the determination of levels of conjugated diene formation in the lipoprotein isolated from plasma. Then, lipoprotein fractions, LDL, sd-LDL and lb-LDL from plasma were added to $\mathrm{CuSO}_{4}$ making final concentration of $5 \mu \mathrm{M}$ and incubated at $37^{\circ} \mathrm{C}$. Durations of $2 \mathrm{~h}$ for LDL and lb-LDL, and $30 \mathrm{~min}$ for sd-LDL were taken for their oxidation. The conjugated diene formation in plasma lipoproteins was quantified by recording absorbance at $234 \mathrm{~nm}$ in a Beckman DU 640 spectrophotometer. Molar extinction coefficient of $2.52 \times 10^{4} \mathrm{M}^{-1} \mathrm{~cm}^{-1}$ was used to calculate conjugated diene formation, and expressed as nmole malondialdehyde (MDA) equivalent per mg LDL, sd-LDL or lb-LDL protein.

Niehaus and Samuelsson [44] described a method to determine MDA content in LDL, sd-LDL and lb-LDL isolated from plasma. In Brief, $2 \mathrm{ml}$ of trichloroacetic acid (15\%)-thiobarbituric acid (0.375\%)-hydrochloric acid $(0.25 \mathrm{~N})$ reagent was added to $0.3 \mathrm{ml}$ of LDL, sd-LDL and lb-LDL, and mixed properly. The solution was kept in a boiling water bath for $15 \mathrm{~min}$. Then flocculent precipitate was removed by centrifugation at $1000 \mathrm{rpm}$ for $10 \mathrm{~min}$. The absorbance of the samples was monitored at $535 \mathrm{~nm}$ against a sample blank. The MDA concentration of the lipoprotein samples was determine by using a molar extinction coefficient $\left(1.56 \times 10^{5} \mathrm{M}^{-1} \mathrm{~cm}^{-1}\right)$.

\section{Protein estimation}

The method of Bradford [45] was used for protein estimation of plasma lipoproteins and liver using bovine serum albumin as standard. Suitable aliquots of the samples were precipitated with $10 \%$ trichloroacetic acid. The obtained protein pellets were dissolved in $0.5 \mathrm{~N}$ $\mathrm{NaOH}$, then suitable portions of the samples were used for protein quantification.

\section{Statistical analysis}

One way analysis of variance (ANOVA) was employed for statistical analysis of the data, then Tukey's Kramer multiple comparison test (GraphPad software Inc., San
Diego, CA, USA) was used. P value $<0.05$ was assumed as significant data.

\section{Results}

\section{GC-MS analysis}

Thirty compounds in methanolic extract (ME) were identified by GC-MS analysis and listed in Table 1. As shown in Table 1, the compounds that were examined in ME, consists of a mixture of terpenes, saturated and unsaturated fatty acids along with certain ester form of compounds. The major compounds were limonene (1.13\%), butylated hydroxytoluene (2.44\%), thymoquinone (2.64\%), palmitic acid (2.77\%), phytol (2.82\%) and glycerol 1-monolinolate (3.99\%). The most dominant compound, an essential fatty acid was identified as linoleic acid (75.00\%). Forty six compounds were identified in volatile oil (VO) extracted from NS seed oil by steam hydrodistillation (Table 2). VO extract consisted of a number of classes of compounds, were various types of nonterpenoid and terpenoid hydrocarbones. The major representative compounds of VO were p-cymene (3.81\%), p-tertbutylpyrocatechol (4.28\%), (+)-trans, trans-5-caranol (7.83\%), 4-terpineol (10.55\%), thymoquinone (13.53\%) and thymol (31.98\%). Thymol represented highest quantity. Other pharmacological minor compounds identified in $\mathrm{VO}$ were isothymol (also called carvacrol), carvone, copaene and limonene oxide. Chromatograms of ME and VO test fractions were given as Additional file 1: Data S1 and Additional file 2: Data S2, respectively.

\section{Body weight, food consumption and weight of liver}

The average body weights of normolipidemic rats were increased from $223 \mathrm{~g}$ to $279 \mathrm{~g}$ after 30-days duration. The body weight of HLP-C group was increased by $20 \%$ when compared to NLP-C rats after giving atherogenic suspension for 30 days. In treated groups with $\mathrm{ME}$ and VO for 30-days duration, the final body weights were significantly reduced by $28 \%$ and $25 \%$, respectively after comparing to HLP-C control group. In the case of the average diet consumption in the all control and treated groups, there were no significant changes observed (data not shown). Average liver weights in HLP-C rats, were increased by $20 \%$ when compared to normolipidemic rats, while in the both treated groups, the liver weights were 5.48 and $5.43 \mathrm{~g}$ with highly significant reduction (Table 3).

\section{Impact on plasma and lipoprotein lipids of rats}

Figure 1 demonstrates that administration of atherogenic suspension to rats resulted in significant increments by $105 \%$ (TG), 168\% (TC), 222\% (non-HDL-C, equivalent to TC minus HDL-C), 105\% (VLDL-C) and 207\% (LDL-C) with substantial decrease by $6 \%(\mathrm{HDL}-\mathrm{C})$ and $13 \%\left(\mathrm{HDL}_{2}-\mathrm{C}\right)$, when compared to the values of normolipidemic rats. Pretreatment with $100 \mathrm{mg}$ ME or $20 \mathrm{mg}$ VO per rat per 
Table 1 Chemical composition of methanolic extract (ME) from Nigella sativa seed oils

\begin{tabular}{|c|c|c|c|}
\hline Peak no. & Compounds & Retention time & Area\% \\
\hline 1 & Limonene & 11.138 & 1.13 \\
\hline 2 & Dihydrocarveol & 13.709 & 0.08 \\
\hline 3 & 4-Carvomenthenol & 15.445 & 0.03 \\
\hline 4 & Thymoquinone & 17.529 & 2.64 \\
\hline 5 & Isothymol & 18.828 & 0.34 \\
\hline 6 & Verbenone & 20.507 & 0.15 \\
\hline 7 & Humulen-(v1) & 21.989 & 0.23 \\
\hline 8 & Butylated hydroxytoluene & 24.380 & 2.44 \\
\hline 9 & Acetovanillone & 25.395 & 0.94 \\
\hline 10 & 3-Methoxy-4,5,6-trimethylphenol & 28.170 & 0.05 \\
\hline 11 & 4-(Methylthio)acetophenone & 28.265 & 0.03 \\
\hline 12 & Myristic acid & 29.861 & 0.06 \\
\hline 13 & Palmitic acid, methyl ester & 33.175 & 0.10 \\
\hline 14 & (Z)6-Pentadecen-1-ol & 34.024 & 0.53 \\
\hline 15 & Palmitic acid & 34.248 & 2.77 \\
\hline 16 & Citronellyl butyrate & 34.376 & 1.05 \\
\hline 17 & Oleic acid, methyl ester & 36.567 & 0.49 \\
\hline 18 & Phytol & 36.747 & 2.82 \\
\hline 19 & Linoleic acid & 38.435 & 75.00 \\
\hline 20 & beta-Monolinolein & 42.187 & 0.45 \\
\hline 21 & Benzedrex & 42.672 & 0.35 \\
\hline 22 & Linoleoyl chloride & 42.968 & 0.58 \\
\hline 23 & $\begin{array}{l}\text { Silane, [1-(5-ethenyltetrahydro-5-methyl-2-furanyl)- } \\
\text { 1-methylethoxy] trimethyl-, trans- }\end{array}$ & 45.655 & 1.74 \\
\hline 24 & Linoleic acid trimethylsilyl esterl & 46.040 & 1.15 \\
\hline 25 & Glycerol 1-monolinolate & 46.443 & 3.99 \\
\hline 26 & Acetylhydroquinone & 51.604 & 0.14 \\
\hline 27 & Squalene & 53.043 & 0.23 \\
\hline 28 & Stigmasterol & 55.298 & 0.11 \\
\hline 29 & gamma-Sitosterol & 56.448 & 0.21 \\
\hline \multirow[t]{2}{*}{30} & Olealdehyde & 58.703 & 0.17 \\
\hline & & & 100.00 \\
\hline
\end{tabular}

day for 30 days in atherogenic suspension fed rats, resulted in significant changes in TG $(-50 \%$ or $-32 \%)$, TC $(-60 \%$ or $-61 \%)$, non-HDL-C $(-69 \%$ or $-67 \%)$, VLDL-C $(-49 \%$ or $-32 \%)$, LDL-C $(-68 \%$ or $-67 \%)$ and HDL-C $(+23 \%$ or $+12 \%), \mathrm{HDL}_{2}-\mathrm{C}(+51 \%$ or $+21 \%)$ and $\mathrm{HDL}_{3}-\mathrm{C}(+11 \%$ or $+8 \%)$, respectively, when compared to the corresponding values in HLP-C rats.

\section{Impact on HDL-linked arylesterase activity and LDL-C:} arylesterase ratio

The data presented in Table 4, was basically obtained from Figure 1. In the lipidemic oxidative stress HLP-C control group, the value of arylesterase was significantly decreased by $21 \%$ coupled with increase in LDL-C:arylesterase ratio by $287 \%$ when compared to values in NLP-C rats. After treatment with the test fractions $\mathrm{ME}$ or VO, substantial restorations in arylesterase activity, and LDL-C:arylesterase ratio in the range of $102 \%-111 \%$, were obtained.

\section{Impact on hepatic 3-hydroxy, 3-methyl glutaryl CoA} reductase activity

A rate limiting enzyme, hepatic 3-hydroxy, 3-methyl glutaryl CoA reductase (HMG-CoA reductase) activity was significantly reduced by $32 \%$ in HLP-C group, when compared to NLP-C value. Further, decline in the values of treated rats with $\mathrm{ME}$ or $\mathrm{VO}$ was observed by $44 \%$ or $42 \%$, respectively, in comparison to NLP-C value (Table 5). 
Table 2 Chemical composition of volatile oil (VO) from Nigella sativa seed oils

\begin{tabular}{|c|c|c|c|}
\hline $\begin{array}{l}\text { Peak } \\
\text { no. }\end{array}$ & Compounds & $\begin{array}{l}\text { Retention } \\
\text { time }\end{array}$ & Area $\%$ \\
\hline 1 & p-Cymene & 11.008 & 3.81 \\
\hline 2 & beta.-Terpineol & 12.233 & 0.48 \\
\hline 3 & Dihydrocarveol & 13.044 & 0.50 \\
\hline 4 & Isothujol & 13.144 & 0.61 \\
\hline 5 & (+)-trans, trans-5-Caranol & 13.744 & 7.83 \\
\hline 6 & 1-Terpinenol & 13.818 & 0.23 \\
\hline 7 & cis-beta-Terpineol & 14.332 & 0.17 \\
\hline 8 & Thujol & 15.029 & 0.14 \\
\hline 9 & 4-Terpineol & 15.507 & 10.55 \\
\hline 10 & p-Cymen-8-ol & 15.639 & 1.62 \\
\hline 11 & Terpineol & 15.830 & 0.38 \\
\hline 12 & Allethrolone & 16.156 & 0.21 \\
\hline 13 & Methoxycitronellal & 16.615 & 0.12 \\
\hline 14 & Carvone & 17.344 & 0.26 \\
\hline 15 & Thymoquinone & 17.535 & 13.53 \\
\hline 16 & trans-Carveol & 17.646 & 0.53 \\
\hline 17 & Linalyl anthranilate & 17.766 & 0.27 \\
\hline 18 & Carvenone & 17.858 & 0.14 \\
\hline 19 & Pichtosin & 18.476 & 0.79 \\
\hline 20 & Thymol & 18.565 & 31.98 \\
\hline 21 & Isothymol & 18.961 & 1.64 \\
\hline 22 & trans,trans-2,4-Decadienal & 19.257 & 0.31 \\
\hline 23 & Copaene & 20.445 & 1.03 \\
\hline 24 & Limonene oxide & 20.892 & 0.34 \\
\hline 25 & (+)-Sativen & 21.892 & 0.12 \\
\hline 26 & Longifolene-(V4) & 22.027 & 7.23 \\
\hline 27 & Methyl 2,5-octadecadiynoate & 23.602 & 0.14 \\
\hline 28 & 2-Tridecanone & 23.750 & 0.63 \\
\hline 29 & beta.-Farnesene & 24.249 & 0.16 \\
\hline 30 & (-)-Isoledene & 24.832 & 0.16 \\
\hline 31 & p-tert-Butylpyrocatechol & 25.421 & 4.28 \\
\hline 32 & beta-Pinene oxide & 26.564 & 0.21 \\
\hline 33 & 1-Cyclododecyl-ethanone & 28.064 & 0.32 \\
\hline 34 & 11-Dodecen-2-one & 28.486 & 0.33 \\
\hline 35 & Epiglobulol & 28.602 & 0.72 \\
\hline 36 & 5-Tetradecen-1-ol, acetate, (Z)- & 29.969 & 0.14 \\
\hline 37 & 2,4,5-Trimethoxy benzoic acid & 30.390 & 0.96 \\
\hline 38 & Palmitic acid, methyl ester & 33.191 & 0.40 \\
\hline 39 & 9-Octadecyne & 34.033 & 0.56 \\
\hline 40 & Rimuen & 34.317 & 1.31 \\
\hline 41 & Cephrol & 34.378 & 1.24 \\
\hline 42 & Preg-4-en-3-one, 12,17-dihydroxy-20-nitrilo- & 35.197 & 0.47 \\
\hline 43 & Methyl linoleate & 36.474 & 0.97 \\
\hline
\end{tabular}

Table 2 Chemical composition of volatile oil (VO) from Nigella sativa seed oils (Continued)

\begin{tabular}{llll}
\hline 44 & Methyl oleate & 36.564 & 0.37 \\
45 & Citronellal & 36.700 & 1.70 \\
46 & Vellerdiol & 37.558 & 0.10 \\
& & & 100.00 \\
\hline
\end{tabular}

Impact on ex vivo and in vitro $\mathrm{Cu}^{2+}$-mediated oxidation of LDL and its subfractions sd-LDL and Ib-LDL and their lag time

As data in Figure 2 demonstrate that maximal conjugated diene (CD) formation from LDL was significantly increased by $299 \%$ in comparison to basal value in NLP-C rats. Feeding of atherogenic suspension to HLP-C rats led to significant increase in ex vivo basal CD formation of LDL, sd-LDL and lb-LDL by $52 \%, 55 \%$ and $20 \%$, respectively in comparison to NLP-C rats. After treatment with $\mathrm{ME}$ or VO, significant reduction in basal CD formation of LDL and its subfractions, sd-LDL and lb-LDL, was seen by $-22 \%$ or $-18 \%,-32 \%$ or $-28 \%$ and $-14 \%$ or $-8 \%$ when compared to the corresponding values in HLP-C rats. In comparison to basal CD values of LDL, sd-LDL and lbLDL in NLP-C, the respective maximal CD values were increased by $299 \%, 305 \%$ and $290 \%$. After treatment with $\mathrm{ME}$ or $\mathrm{VO}$, a substantial decrease in $\mathrm{CD}$ product of LDL, sd-LDL and lb-LDL by $32 \%$ or $22 \%, 40 \%$ or $30 \%$ and $13 \%$ or $8 \%$, respectively was observed when compared to atherogenic suspension fed control rats. In the case of lag phase of LDL, sd-LDL and lb-LDL lipoproteins, a substantial decrease by $41 \%$ (53 $\mathrm{min}$ ), $35 \%$ (11 min) and 29\% (36) in the stressed control group of rats was seen, when compared to the normolipidemic control rats. Similar to $C D$ values, lag phase of the treated groups was significantly restored and increased close to the corresponding normal values in NLP-C rats (Figure 2).

Impact on $\mathrm{Cu}^{2+}$-induced generation of malondialdehyde in plasma $\mathrm{LDL}$, sd-LDL and Ib-LDL

As in Table 6, basal malondialdehyde (MDA) formation of LDL, sd-LDL and lb-LDL was substantially increased by $106 \%$ (6.47 nmole/mg protein), $71 \%$ (8.05 nmole/mg protein) and $61 \%$ (6.18 nmole/mg protein), respectively in HLP-C rats when compared to the corresponding values in NLP-C rats after 30-days duration. In comparison to MDA values of HLP-C rats, the maximal MDA values of LDL, sd-LDL and lb-LDL were significantly increased by $81 \%, 66 \%$ and $32 \%$, respectively. After treatment with the test fractions, ME or $\mathrm{VO}$ a significant basal restoration close to normal values was observed as $78 \%$ or $65 \%, 81 \%$ or $68 \%$, and $80 \%$ or $72 \%$ and maximal restoration as $83 \%$ or $72 \%, 84 \%$ or $70 \%$ and $93 \%$ or $87 \%$, respectively. 
Table 3 Average body and liver weights of rats during $\mathbf{3 0}$ days of treatment with ME and VO fractions

\begin{tabular}{lccr}
\hline Group & \multicolumn{2}{c}{ Body weight per rat $(\mathbf{g})$} & Liver weight per rat (g) \\
\cline { 2 - 3 } & Prior to treatment & Post treatment & $5.44 \pm 0.22$ \\
\hline NLP-C & $233.30 \pm 20.8$ & $278.60 \pm 16.72$ & $6.51 \pm 0.01(+19.65 \%)^{a}$ \\
HLP-C & $22.00 \pm 14.71$ & $334.50 \pm 14.50(+20.06 \%)^{a}$ & $5.48 \pm 0.03(-15.82 \%)^{b}$ \\
HLP- ME & $221.25 \pm 13.14$ & $240.25 \pm 9.74(-28.18 \%)^{b}$ & $5.43 \pm 0.07(-16.59 \%)^{b}$ \\
HLP-VO & $220.00 \pm 10.80$ & $250.75 \pm 4.85(-25.04 \%)^{b}$ & \\
\hline
\end{tabular}

Each value is mean \pm SD from 4 rats in each group except NLP $(n=5)$. NLP-C, normolipidemic control fed $1 \mathrm{ml}$ saline/rat/day; HLP-C, hyperlipidemic control given $1 \mathrm{ml}$ of saline before the feeding of $1 \mathrm{ml}$ suspension containing $5 \mathrm{mg}$ cholesterol, $30 \mathrm{mg}$ coconut oil and $2.5 \mathrm{mg}$ cholic acid /rat/day; whereas rats in HLP-ME, HLP-VO were fed $1 \mathrm{ml}$ of $100 \mathrm{mg} \mathrm{ME}, 20 \mathrm{mg}$ VO prior to administration of $1 \mathrm{ml}$ of the above atherogenic suspension /rat/day for 30 days. Note: All the values in NLP-C and HLP-C control groups in this manuscript were already presented in our previously published article [37]. The values were statistically significant from NLP-C at ${ }^{a} p<0.05$ and HLP-C at ${ }^{b} p<0.05$.

\section{Discussion}

Methanolic extract (ME) and volatile oil (VO) extracted from NS seed oil containing different phenolic, and terpenoid compounds along with other pharmaceutical compounds exert their effects on hypercholesterolemic rats through multiple mechanisms. In this study, we investigated hypolipidemic and antioxidant actions of $\mathrm{ME}$ and VO in atherogenic suspension fed rats. Administration of atherogenic suspension to rats containing $30 \mathrm{mg}$ coconut oil, $5 \mathrm{mg}$ cholesterol and $2.5 \mathrm{mg}$ cholic acid per day/rat for

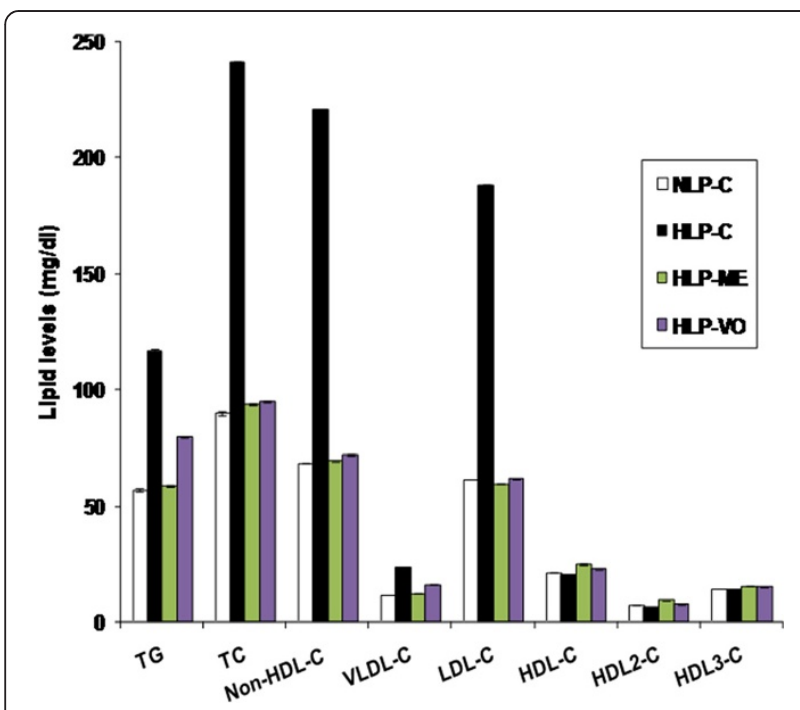

Figure 1 Protective effects of ME and VO extracts of NS oil on plasma TG, TC, non-HDL-C (TC-HDL-C), VLD-C, LDL-C, HDL-C, $\mathrm{HDL}_{2}-\mathrm{C}$ and $\mathrm{HDL}_{3}-\mathrm{C}$ subfractions. NLP-C, normolipidemic control fed $1 \mathrm{ml}$ saline/rat/day; HLP-C, hyperlipidemic control given $1 \mathrm{ml}$ of saline before the feeding of $1 \mathrm{ml}$ suspension containing $5 \mathrm{mg}$ cholesterol, $30 \mathrm{mg}$ coconut oil and $2.5 \mathrm{mg}$ cholic acid/rat/day; whereas rats in HLP-ME and HLP-VO were fed $1 \mathrm{ml}$ of $100 \mathrm{mg}$ ME or $20 \mathrm{mg} \mathrm{VO}$, prior to administration of $1 \mathrm{ml}$ of the above atherogenic suspension/rat/day for 30 days. Each value represents mean \pm SD from pooled plasma samples in each group. The values of HLP-C control groups were statistically significant from NLP-C groups $(p<0.05)$, the values of treated groups were significantly different from HLP-C groups $(p<0.05)$, and the values between the treated groups were also significant $(p<0.05)$.
30 days exerted significant increments in the body and liver weights in HLP-C group of rats. Such gains in the weights were apparently owing to the deposition of lipid contents in the body, especially in the liver. Feeding of cholic acid, a component of atherogenic suspension helped in the intestinal absorption of the both cholesterol and fatty acids. This could be one of the reasonable factors that could explain the weight gains in the average body as well as the liver of the rats. Thirty day feeding of ME and VO suspensions significantly reduced the body weight along with liver weight, possibly by reducing the accumulation of fatty acids in adipose tissue of the treated rats, when compared to normolipidemic rats (Table 3).

As seen in HDL-C rats (Figure 1), increases in plasma TG and VLDL-C concentrations may be due to high production of VLDL with decreased clearance rate of TG as reported in hepatic $\beta$-VLDL secretion in cholesterol fed rabbits [46]. Presence of high concentration of plasma VLDL-C it may also lead to decreased production of antiatherogenic HDL because of the low availability of phospholipid remnants for its formation from VLDL and decline in the activity of lecithin cholesterol:acyltransferase as reported in several published reports. Treatment with the test fractions to the rats led to significant reduction in the levels of plasma TG, TC, VLDL-C, LDL-C and non-HDL-C, and antiatherogenic

Table 4 Effect of methanolic extract (ME) and volatile oil (VO) fractions on plasma arylesterase activity and the ratio of LDL-C and arylesterase activity in rats after 30 days of treatment

\begin{tabular}{lcc}
\hline Group & $\begin{array}{c}\text { Arylesterase activity } \\
(\text { nmole/min/ml) }\end{array}$ & LDL-C $^{+}$:arylesterase activity \\
\hline NLP-C & $665.15 \pm 4.60$ & $0.9220 \times 10^{-3} \pm 0.180 \times 10^{-5}$ \\
HLP-C & $527.34 \pm 4.73(-20.71 \%)^{\mathrm{a}}$ & $3.5682 \times 10^{-3} \pm 0.225 \times 10^{-5}(+287.00 \%)^{\mathrm{a}}$ \\
HLP- ME & $711.61 \pm 4.70(+34.94 \%)^{\mathrm{b}}$ & $0.8338 \times 10^{-3} \pm 0.170 \times 10^{-5}(-76.63 \%)^{\mathrm{b}}$ \\
HLP-VO & $680.57 \pm 5.20(+29.05 \%)^{\mathrm{b}}$ & $0.9020 \times 10^{-3} \pm 0.180 \times 10^{-5}(-74.72 \%)^{\mathrm{b}}$
\end{tabular}

${ }^{\dagger} \mathrm{LDL}-\mathrm{C}(\mathrm{mg} / \mathrm{ml})$ values are taken from Figure 1. Each value represents mean \pm $S D$ from pooled plasma samples in each group. The values were statistically significant from NLP-C at ${ }^{a} p<0.05$ and HLP-C at ${ }^{b} p<0.05$, and the values between the treated groups were also significant $(p<0.05)$. 
Table 5 In vivo modulation effect of ME and VO fractions on hepatic HMG-CoA reductase activity in atherogenic suspension fed rats

\begin{tabular}{lc}
\hline Group & HMG-CoA reductase activity† \\
\hline NLP-C & $28.90 \pm 0.04$ \\
HLP-C & $19.72 \pm 0.07( \pm 31.76 \%)^{a}$ \\
HLP-ME & $16.33 \pm 0.06(-43.49 \%)^{a}$ \\
HLP-VO & $16.74 \pm 0.05(-42.07 \%)^{a}$
\end{tabular}

† Its activity is expressed as percent of nmoles of HMG-CoA to mevalonate formed per mg liver protein. Each value represents mean \pm SD from pooled liver samples in each group. The values were highly significant from NLP-C at $a_{p}<0.001$, and the values between the treated groups were also statistically significant $(p<0.05)$

HDL-C along with its subfractions were significantly increased, and finally restoration of these altered lipoproteins were gained to near or above normal values. In this observation of results, ME had proven itself as higher potential agent than VO. Arylesterase is an important plasma HDL-linked antioxidant enzyme, protecting LDL from oxidative modification. LDL-C:arylesterase ratio, a good marker is used in hyperlipidemia. Thus, it is clear from the discussion that in lipidemic oxidative stress arylesterase, and LDL-C:arylesterase ratio were

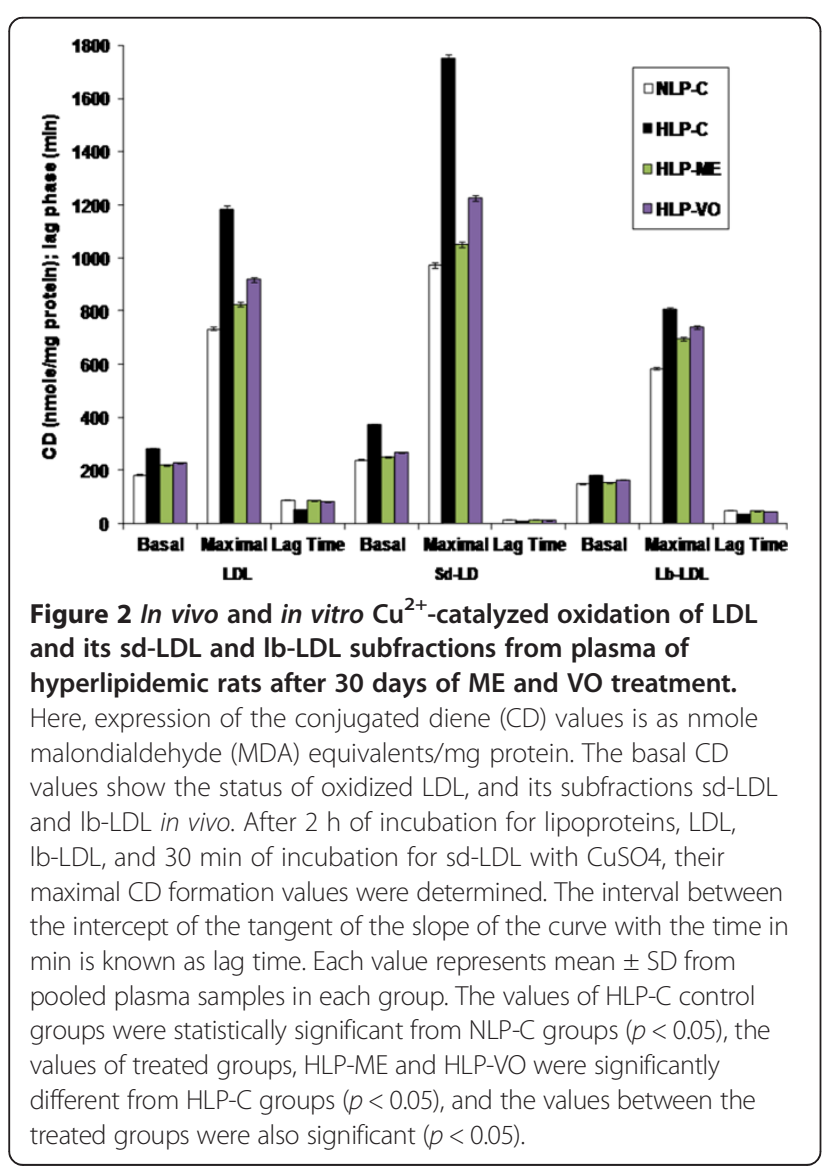

substantially changed apparently due to prooxidant effect against oxidation of LDL and ultimately malondialdehyde (MDA/TBARS) production. It is also evident from the discussion; decrease in arylesterase was consistent with HDL-C decrease and vice versa. Treatment with ME or $\mathrm{VO}$ to rats, restoration of arylesterase and HDL-C along with its subfractions was higher to normal values, and therefore arylesterase may play an important role in the antioxidation, anti-inflamatory or antiatherosclerotic properties [47].

An important rate-limiting enzyme, HMG-CoA reductase was suppressed in HLP-C rats by feeding atherogenic suspension (Table 5). Activity of HMG-CoA reductase in HLP-ME and HLP-VO rats was further reduced that apparently involved two related mechanisms as reported by Al-Naqeep et al. [48], in thymoquinone rich fraction treated rats: suppression of hepatic HMG-CoA reductase mRNA expression and increment in LDL receptor gene. Such regulations could clearly explain the hypolipidemic properties of $\mathrm{ME}$ and $\mathrm{VO}$ test factions that contained thymoquinone $(2.64 \%)$ and (13.53\%), respectively. Other compounds present in these test fractions may exert independently or synergistically (Tables 1 and 2) on lipidemic oxidative stress in rats. Atherogenic suspension induced oxidative stress and increased susceptibility of lipoproteins and other membrane lipids to oxidation in rats. Oxidative stress was result from equilibrium loss between antioxidant and oxidant systems. As in Figure 1 and Table 4, it is clearly evident that antioxidant HDL-linked arylesterase was significantly decreased and increased cholesterol content of LDL and LDL-C:arylesterase ratio in HLP-C rats compared to NLP-C rats. Increased LDL-C, low HDL-C and elevated TG levels (lipid triad) coupled with decrease in HDL-linked arylesterase in lipidemic oxidative stress rats are reflected in CD and MDA productions in LDL and its subfractions sd-LDL and lb-LDL. Sd-LDL subfraction of LDL is assumed comparatively higher inducer than lb-LDL for proatherogenic process in the vessel wall of humans with hyperlipidemia, similar to atherogenic lipoprotein phenotype pattern B subjects [49,50]. Because of several oxidizable properties were attributed to it, such as reduced content of free cholesterol and antioxidant, increased amount of more oxidizable polyunsaturated fatty acids [50,51]. Thus, more conjugated diene (CD) formation in sd-LDL than lb-LDL was seen in the both basal and maximal CD productions in lipidemic induced hyperlipidemic rats. Further, small lag time of sd-LDL of HLP-C rats compared to respective values in $\mathrm{lb}-\mathrm{LDL}$ shows higher prone to oxidative damage. This could also reflect in increased $\mathrm{Cu}^{2+}$-induced ex vivo basal and maximal MDA production.

Administration of lipidemic stress in rats with the test fractions, ME and VO resulted in the restoration of $C D$ and MDA levels of LDL and its subspecies sd-LDL and lb-LDL to an average value of $85 \%$, when compared to 
Table 6 In vivo and in vitro $\mathrm{Cu}^{2+}$-induced generation of malondialdehyde (MDA) in plasma LDL, sd-LDL and Ib-LDL from rats after 30 days of methanolic extract (ME) and volatile oil (VO) treatment

\begin{tabular}{|c|c|c|c|c|c|c|}
\hline \multirow[t]{3}{*}{ Group } & \multicolumn{6}{|c|}{ Malondialdehyde $^{\dagger}$} \\
\hline & \multicolumn{2}{|c|}{ LDL } & \multicolumn{2}{|c|}{ Sd-LDL } & \multicolumn{2}{|c|}{ Lb-LDL } \\
\hline & Basal & Maximal & Basal & Maximal & Basal & Maximal \\
\hline NLP-C & $3.14 \pm 0.05$ & $26.69 \pm 0.11(+750.00 \%)$ & $4.71 \pm 0.02$ & $33.02 \pm 0.09(+601.06 \%)$ & $3.83 \pm 0.08$ & $24.73 \pm 0.05(+549.69 \%)$ \\
\hline HLP-C & $6.47 \pm 0.04(+106.05 \%)^{\mathrm{a}}$ & $48.40 \pm 0.09(+81.31 \%)^{a}$ & $8.05 \pm 0.06(+70.91 \%)^{a}$ & $55.60 \pm 0.05(+66.38 \%)^{a}$ & $6.18 \pm 0.07(+61.35 \%)^{a}$ & $32.68 \pm 0.06(+32.14 \%)^{a}$ \\
\hline HLP-ME & $4.05 \pm 0.08(-37.40 \%)^{b}$ & $32.02 \pm 0.06(-33.84 \%)^{b}$ & $5.78 \pm 0.05(-28.19 \%)^{b}$ & $39.37 \pm 0.06(-29.19 \%)^{b}$ & $4.79 \pm 0.09(-22.49 \%)^{b}$ & $26.73 \pm 0.07(-18.20 \%)^{b}$ \\
\hline HLP-VO & $4.80 \pm 0.06(-25.81 \%)^{b}$ & $36.98 \pm 0.03(-23.59 \%)^{b}$ & $6.92 \pm 0.05(-14.03 \%)^{b}$ & $47.08 \pm 0.07(-15.32 \%)^{b}$ & $5.30 \pm 0.04(-14.23 \%)^{b}$ & $28.30 \pm 0.05(-13.40 \%)^{b}$ \\
\hline
\end{tabular}

${ }^{\dagger}$ The values are expressed as nmole/mg protein. The basal MDA values in the table show the in vivo status of oxidized LDL, and its subfractions sd-LDL or Ib-LDL. In the case of maximal MDA values of the lipoproteins ( $\mathrm{LDL}$ and $\mathrm{lb}$-LDL oxidation) were achieved after $2 \mathrm{~h}$ of incubation with CuSO $\mathrm{C}_{4}$, whereas for sd-LDL maximal values were obtained after 30 min. Each value represents mean \pm SD from LDL, sd-LDL and Ib-LDL subfractions, isolated from pooled plasma samples in each group. The values were statistically significant from NLP-C at ${ }^{a} p<0.05$ and HLP-C at ${ }^{b} p<0.05$, and the values between the treated groups were also significant $(p<0.05)$.

the respective values in normolipidemic control rats, apparently through radical scavenging properties of the test factions $[14,18]$. Overall, hypolipidemic and antioxidant properties of ME were greater than VO in these stressed rats. Such effects of the test fractions are deciphered to indicate that the ME and VO strongly inhibit peroxidation of the molecules or resulted in elimination of the reactive toxic intermediates initiated by free radicals, thus preventing or delaying the cell damage. The above antioxidant and hypolipidemic actions of these test fractions are strongly supported by our previously published reports in which thymoquinone and limonene showed such strong effects $[19,37]$. However, the combined data from in vivo and in vitro studies strongly suggest that overall alleviation of ROS related disorders is due to highly effective amelioration of elevated levels of plasma atherogenic TG/TG rich lipoproteins, highly oxidizable and proatherogenic sd-LDL subspecies of LDL and low levels of atheroprotective HDL-C, i.e., lipid triad, coupled with a strong inhibition of in vivo/ex vivo and in vitro lipid peroxidation through efficient free radical scavenging properties of the test fractions and compounds in the order ME extract $>$ pure thymoquinone $>\mathrm{VO}$ extract $>$ pure limonene.

The above discussion conferred that the principal active constituents thymoquinone, limonene were identified as antioxidant as well as hypolipidemic agents along with linoleic acid ( $\omega-6$ fatty acid) that shows hypocholesterolemic activity in the presence of saturated fatty acid, palmitic acid in the ratio of 27:1, respectively. Thus, a combination of thymoquinone, limonene and linoleic acid in association with plamitic acid may be called as compound triad is highly effective in lipidemic oxidative stress when treated with ME. VO represented three related active compounds namely, thymol, thymoquinone and isothymol as antioxidants, they may be termed as thyme triad. VO, despite of being quite effective in alleviation of hyperlipidemia it lacks essential fatty acid and reduced form of limonene is comparatively less active than ME. It is apparently due to that limonene itself as hypolipidemic, and linoleic acid in the presence of palmitic acid show additive effects of thymoquinone for removal of lipid by suppression of HMG-CoA reductase activity and increased levels of LDL receptor in agreement with Al-Naqeep et al. [48] consequently resulted in significant decrease in oxidative stress in ME treated rats. Thus, it is clear that most abundant compound, linoleic acid in ME (Table 1) has a significant role in controlling the lipid levels and oxidative stress than VO. Because of VO, it showed lack of reduced form of limonene and absence of linoleic acid. However, the both test fractions likely share common mechanisms by suppressing HMG-CoA reductase activity, increased levels of LDL receptor [48], and free radical scavenging activity, other active constituents show independent or synergistic effects to alleviate lipidemic oxidative stress.

\section{Conclusions}

The test fractions, ME and VO having thymoquinone common to the both extracts, and other pharmaceutical important compounds effectively ameliorated/normalized all the CVD risk parameters through modulation of HMGCoA reductase activity, levels of LDL receptor and antioxidant mechanisms in atherogenic suspension fed rats. Thus, $\mathrm{ME}$ and VO both can be used as hypolipidemic, antioxidant, antiperoxidative and antiatherogenic agents in CVD and its complications. In future, it will need to elucidate the mechanisms of the principal effective compounds and their additive role of different combinations of the known compounds by GC-MS analysis of ME and VO to exert their lipid lowering and antioxidant actions, including the vascular status such as histopathological and plaque load studies after thorough evaluation study of toxic effect.

\section{Additional files}

Additional file 1: Data S1. Chromatogram of methanolic extract (ME) obtained from Nigella sativa seed oil. The each corresponding peaks were identified as given in Table 1. 
Additional file 2: Data S2. Chromatogram of volatile oil (VO) extracted from Nigella sativa seed oil. The each corresponding peaks were identified as given in Table 2

\section{Abbreviations}

CD: Conjugated diene; CHD: Coronary heart disease; CVD: Cardiovascular disease; GC-MS: Gas chromatography-mass spectrometry; HDL-C: High density lipoprotein cholesterol; HLP-C: Hyperlipidemic control; HLPME: Hyperlipidemic ME; HLP-VO: Hyperlipidemic VO; HMG-CoA: 3-Hydroxy-3methylglutaryl coenzyme A; Lb-LDL: Large buoyant-LDL; LDL-C: Low density lipoprotein cholesterol; MDA: Malondialdehyde; NLP-C: Normolipidemic control; NS: Nigella sativa; ME: Methanolic extract; ROS: Reactive oxygen species; Sd-LDL: Small dense-LDL; TBARS: Thiobarbituric acid reactive substances; TC: Total cholesterol; TG: Triglycerides; VLDL-C: Very low density lipoprotein cholesterol; VO: Volatile oil.

\section{Competing interests}

The authors declare that they have no competing interests.

\section{Authors' contributions}

SA carried out the experiments, prepared the Tables, Figures, performed the statistical analysis, and wrote the manuscript. ZHB participated in the interpretation of the data and writing the manuscript. Both authors read and approved the final manuscript.

\section{Acknowledgements}

The authors express their gratitude to Dr. Ajay Kumar, Advanced Instrumentation Research Facility (AIRF), Jawaharlal Nehru University, New Delhi, Dr. Muhammad Anas, Department of Pharmacology, A.K. Tibbiya College, Aligarh Muslim University, Aligarh for their analytical support of GC-MS analysis, and University Grants Commission for predoctoral fellowship.

Received: 9 April 2013 Accepted: 10 June 2013

Published: 13 June 2013

\section{References}

1. WHO: Cardiovascular disease; Fact sheet No317. Geneva, Switzerland; 2009 http://www.who.int/mediacentre/factsheets/fs317/en/print.html.

2. WHO: Diet, nutrition and prevention of chronic diseases. Report of a joint WHO/FAO expert consultation. Avenue Appia, Geneva: Marketing and Dissemination, World Health Organization (Switzerland). World Health Organ Tech Rep Ser. 2003, 916.

3. Stehbens W: An appraisal of cholesterol feeding in experimental atherosclerosis. Prog Cardiovasc Dis 1986, 29:107-128.

4. Bulur H, Ozdemirler G, Oz B, Toker G, Ozturk M, Uysal M: High cholesterol diet supplemented with sunflower seed oil but not olive oil stimulates lipid peroxidation in plasma, liver, and aorta of rats. J Nutr Biochem 1995, 6:547-550.

5. Halliwell B: Mechanisms involved in the generation of free radicals. Pathol Biol (Paris) 1996, 44:6-13.

6. Stamler J, Wentforth D, Neaton JD: Is the relationship between serum cholesterol and risk of premature death from coronary heart disease continuous and graded? Findings in 356,222 primary screens of the Multiple Risk Factor Intervention Trial (MRFIT). J Am Med Assoc 1986, 256:2823-2828.

7. Bjornheded T, Babyi A, Bodjers G, Wilkund O: Accumulation of lipoprotein fraction and subfractions in the arterial wall, determined in an in vitro perfusion system. Atherosclerosis 1996, 123:43-56.

8. Chapman MJ, Guerin M, Bruckert E: Atherogenic, dense lowdensity-lipoproteins. Pathophysiology and new therapeutic approaches. Eur Heart J 1998, 19(suppl A):A24-A30.

9. Austin MA, Mykkanen L, Kuusisto J, Edwards KL, Nelson C, Haffner SM Pyorala K, Laakso M: Prospective study of small dense LDLs as a risk factor for non-insulin dependent diabetes mellitus in elderly men and women. Circulation 1995, 92:1770-1778

10. Hirano T, Ito Y, Koba S, Toyoda M, Ikejiri A, Saegusa H, Yamazaki J, Yoshino G: Clinical significance of small dense low-density lipoprotein cholesterol levels determined by the simple precipitation method. Arterioscler Thromb Vasc Biol 2004, 24:558-563.
11. Koba S, Hirano T, Ito Y, Tsunoda F, Yokota Y, Ban Y, Iso Y, Suzuki H, Katagiri $\mathrm{T}$ : Significance of small low-density lipoprotein-cholesterol concentration in relation to the severity of coronary heart disease. Athersclerosis 2006, 189:206-214.

12. Annie A, Kurup PA: Dietary carbohydrates and regulation of the activity of HMG-CoA reductase and cholesterol metabolism. Indian J Biochem Biophys 1986, 23:28-31.

13. Manach C, Mazur A, Scalbert A: Polyphenols and prevention of cardiovascular diseases. Curr Opin Lipidol 2005, 16:77-84.

14. Yu LL, Zhou KK, Parry J: Antioxidant properties of cold-pressed black caraway, carrot, cranberry, and hemp seed oils. Food Chem 2005, 91:723-729.

15. Shahidi F, Chandrasekara A, Zhong Y: Bioactive phytochemicals in vegetables. In Handbook of vegetables and vegetable processing. Edited by Singha NK. State Avenue, Ames (USA). lowa: John Wiley \& Sons Publishing Ltd; 2011:125-158.

16. Shahidi F, Naczk M: Phenolics in food and nutraceuticals. Boca Raton, FL: CRC Press; 2004:1-82

17. Bors W, Heller W, Michel C, Saran M: Flavonoids as antioxidants: determination of radical scavenging efficiencies. Meth Enzymol 1990, 186:343-355.

18. Burits M, Bucar F: Antioxidant activity of Nigella sativa essential oil. Phytother Res 2000, 14:323-328

19. Ahmad S, Beg ZH: Alleviation of plasma, erythrocyte and liver lipidemic-oxidative stress by thymoquinone and limonene in atherogenic suspension fed rats. J Funct Foods 2013, 5:251-259.

20. Yanishlieva NV, Marinova EM, Gordon MH, Raneva VG: Antioxidant activity and mechanism of action of thymol and carvacrol in two lipid systems. Food Chem 1999, 64:59-66.

21. Spady DK, Woollett LA, Dietschy JM: Regulation of plasma LDL-cholesterol levels by dietary cholesterol and fatty acids. Annu Rev Nutr 1993, 13:355-381.

22. Champe PC, Harvey RA, Ferrier DR: Lipincott's Illustrated Reviews: Biochemistry. 4th edition. New Delhi: Wolters Kluwer (India) Pvt. Ltd; 2008. chapter 27.

23. Stampfer MJ, Krauss RM, Ma J, Blanche PJ, Holl LG, Sacks FM, Hennekens CH A prospective study of triglyceride level, low-density lipoprotein particle diameter, and risk of myocardial infarction. J Am Med Assoc 1996, 276:882-888.

24. Austin MA, Hokanson JE, Edwards KL: Hypertriglyceridemia as a cardiovascular risk factor. Am J Cardiol 1998, 81:7B-12B.

25. Gordon DJ, Probstfield JL, Garrison RJ, Neaton JD, Castelli WP, Knoke JD, Jacobs DR Jr, Bangdiwala S, Tyroler HA: High-density lipoprotein cholesterol and cardiovascular-disease-4 prospective American studies. Circulation 1989, 79:8-15.

26. Frick $\mathrm{MH}$, Elo $\mathrm{O}$, Haapa $\mathrm{K}$, et al: Helsinki heart study: primary-prevention trial with gemfibrozil in middle-aged men with dyslipidemia. Safety of treatment, changes in risk factors, and incidence of coronary heart disease. N Engl J Med 1987, 317:1237-1245.

27. Machhi JP, Shah NN: Study of antiatherosclerotic activity of polyherba preparation using rat as an experimental animal model. IJPSR 2012, 3:4010-4018.

28. Zhao J, Quan-Xin Y, Kong W, Hai-Cheng G, Sun B, Ya-Qin X, Li-Qun R: The urotensin II receptor antagonist, urantide, protects against atherosclerosis in rats. Exp Ther Med 2013, 5:1765-1769.

29. Kanter M, Coskun O, Uysal $\mathrm{H}$ : The antioxidative and antihistaminic effect of Nigella sativa and its major constituent, thymoquinone on ethanol-induced gastric mucosal damage. Arch Toxicol 2006, 80:217-224.

30. Houcher Z, Boudiaf K, Benboubetra M, Houcher B: Effects of methanolic extract and commercial oil of Nigella sativa L. on blood glucose and antioxidant capacity in alloxan-induced diabetic rats. Pteridines 2007, 18:8-18.

31. Kanter M: Effects of Nigella sativa and its major constituent thymoquinone on sciatic nerves in experimental diabetic neuropathy. Neurochem Res 2008, 33:87-96

32. Vahdati-Mashhadian N, Rakhshandeh H, Omidi A: An investigation on LD50 and subacute hepatic toxicity of Nigella sativa seed extracts in mice. Pharmazie 2005, 60:544-547.

33. Kanter M, Meral I, Yener Z, Ozbek H, Demir H: Partial regeneration proliferation of the beta-cells in the islets of Langerhans by Nigella sativa L. in streptozotocin-induced diabetic rats. Tohoku J Exp Med 2003, 201:213-219.

34. Wieland $H$, Seidel D: A simple method for precipitation of low-density lipoproteins. J Lipid Res 1989, 24:904-909. 
35. Bachorik PS, Albers JJ: Precipitation methods for quantification of lipoproteins. Meth Enzymol 1986, 129:78-100.

36. Kostner GM: Letter: Enzymatic determination of cholesterol in high-density lipoprotein fractions prepared by polyanion precipitation. Clin Chem 1976, 22:695.

37. Ahmad S, Beg ZH: Hypolipidemic and antioxidant activities of thymoquinone and limonene in atherogenic suspension fed rats. Food Chem 2013, 138:1116-1124.

38. Trinder P: Determination of glucose in blood using glucose oxidase with an alternative oxygen receptor. Ann Clin Biochem 1969, 6:24-27.

39. Rao AV, Ramakrishnan S: Indirect assessment of hydroxymethylglutaryl-CoA reductase (NADPH) activity in liver tissue. Clin Chem 1975, 21:1523-1525.

40. Ayub A, Mackness MI, Arrol S, Mackness B, Patel J, Durrington PN: Serum paraoxonase after myocardial infarction. Arterioscler Thromb Vasc Biol 1999, 19:330-335.

41. La Du BN, Eckerson HW: The polymorphic paraoxonase/arylesterase isozymes of human serum. Fed Proc 1984, 43:2338-2341.

42. Esterbauer H, Striegel G, Puhl H, Oberreither S, Rotheneder M, el-Saadani M, Jürgens $\mathrm{G}$ : The role of vitamin $\mathrm{E}$ and carotenoids in preventing oxidation of low-density lipoproteins. Ann Ny Acad Sci 1989, 570:254-267.

43. Esterbauer H, Gebicki J, Puhl H, Jugens G: The role of lipid peroxidation and antioxidants in oxidative modification of LDL. Free Radical Bio Med 1992, 13:341-390.

44. Niehaus WG, Samuelsson B: Formation of malondialdehyde from phospholipids arachidonate during microsomal lipid peroxidation. Eur J Biochem 1968, 6:126-130.

45. Bradford MM: A rapid and sensitive method for the quantification of microgram quantities of protein utilizing the principle of protein-dye binding. Anal Biochem 1976, 72:248-254.

46. Mackinnon AM, Savage J, Gibson RA, Barter PJX: Secretion of cholesteryl ester enriched very low density lipoproteins by the liver of cholesterol-fed rabbits. Atherosclerosis 1985, 54:145-155.

47. Aviram M, Rosenblat M, Bisgaier CL, Newton RS, Primo-Parmo SL, La Du BN: Paraoxonase inhibits high-density lipoprotein oxidation and preserves its functions. A possible peroxidative role for paraoxonase. J Clin Invest 1998, 101:1581-1590.

48. Al-Naqeep G, Ismail M, Yazan LS: Effects of thymoquinone rich fraction and thymoquinone on plasma lipoprotein levels and hepatic low density lipoprotein receptor and 3-hydroxy-3-methylglutaryl coenzyme A reductase genes expression. J Funct Foods 2009, 1:298-303.

49. Chait A, Brazg RL, Tribble DL, Krauss RM: Susceptibility of small, dense, low-density lipoproteins to oxidative modification in subjects with the atherogenic lipoprotein phenotype, pattern B. Am J Med 1993, 94:350-356.

50. Tribble DL, Rizzo M, Chait A, Diane ML, Blanche PJ, Krauss RM: Enhanced oxidative susceptibility and reduced antioxidant content of metabolic precursor of small, dense low-density lipoproteins. Am J Med 2001, 110:103-110.

51. de Graaf J, Hak-Lemmers HL, Hectors MP, Demacker PN, Hendriks JC, Stalenhoef AF: Enhanced susceptibility to in vitro oxidation of the dense low-density lipoprotein subfraction in healthy subjects. Arterioscler Thromb Vasc Biol 1991, 11:298-306.

\section{doi:10.1186/1476-511X-12-86}

Cite this article as: Ahmad and Beg: Elucidation of mechanisms of actions of thymoquinone-enriched methanolic and volatile oil extracts from Nigella sativa against cardiovascular risk parameters in experimental hyperlipidemia. Lipids in Health and Disease 2013 12:86.

\section{Submit your next manuscript to BioMed Central and take full advantage of:}

- Convenient online submission

- Thorough peer review

- No space constraints or color figure charges

- Immediate publication on acceptance

- Inclusion in PubMed, CAS, Scopus and Google Scholar

- Research which is freely available for redistribution 